\title{
MODIFIED NLM MODEL FOR DESPECKLING ULTRASOUND IMAGES USING FCM CLUSTERING BASED PRE CLASSIFICATION AND RIBM
}

\author{
K.M. Prabusankarlal ${ }^{1}$, P. Thirumoorthy ${ }^{2}$, R. Manavalan $^{\mathbf{3}}$ and R. Sivaranjani $^{\mathbf{4}}$ \\ ${ }^{I}$ Department of Electronics and Communication, K.S. Rangasamy College of Arts and Science, India \\ ${ }^{2}$ Department of Electronics and Communication, Government Arts College, Dharmapuri, India \\ ${ }^{3}$ Department of Information Technology, Arignar Anna Government Arts College, India \\ ${ }^{4}$ Department of Mathematics, K.S.R. College of Arts and Science for Women, India
}

\begin{abstract}
Speckle noise is an inherent characteristic of ultrasound which reduces the classification accuracy of computer aided diagnosis (CAD) systems. A modified non local means (NLM) filter for despeckling ultrasound images is proposed in this article. The proposed NLM model utilizes a preclassification method in which the feature vectors of the input image are constructed using moment invariants and then they are clustered using fuzzy c means (FCM) algorithm. The rotationally invariant block matching (RIBM) algorithm is applied among the blocks within each cluster instead of the entire image. This intra cluster block matching reduces computational complexity of NLM process without the elimination of any pixel candidate. Further, the rotationally invariant moment distance measure improves the noise reduction performance of the algorithm by increasing the chance of getting more similar candidates for NLM process. Extensive experiments are conducted using synthetic images, phantom images and ultrasound images. The method is comparatively evaluated with other denoising methods using statistical parameters such as MSE, PSNR, SSIM, EPI and ENL. The quantitative results suggested that the proposed method outperforms other four state of the art methods in despeckling and preservation of image details.
\end{abstract}

Keywords:

Speckle Noise, Non Local Means, Fuzzy C Means, Ultrasound, CAD Systems

\section{INTRODUCTION}

Ultrasound has become an important imaging modality in diagnosing cancer [1]. Many computer aided diagnosis (CAD) systems have been developed to reduce the operator dependence and to increase the sensitivity and specificity of clinical diagnosis [2]. The important deficiency of ultrasound is the poor image quality due to the speckle noise. The existence of the speckle degrades the image quality and impacts the diagnosis accuracy [3], [4]. The objective of image denoising task is to suppress the speckle noise while retaining the signal features as much as possible in order to increase the efficiency, accuracy and productivity [5].

An accurate model of speckle noise formation is required for the development of a despeckling algorithm. Many statistical models were developed to describe speckle noise, but a universally accepted noise model is not available yet. However, a generalized model of the speckle noise [6] is given,

$$
g(n, m)=f(n, m) u(n, m)+\xi(n, m)
$$

where, $g(n, m)$ is the observed image, $f(n, m)$ is the original image. The multiplicative component of the speckle noise is $u(n, m)$ and the additive component is $\xi(n, m)$. The indices $n$ and $m$ denote the axial and lateral indices of the image respectively. In most of the despeckling methods, only the multiplicative component $u(n, m)$ is considered and hence the model is simplified by neglecting the additive component. The simplified model is given as [7],

$$
g(n, m) \simeq f(n, m) u(n, m)
$$

In the literature of breast ultrasound, the most preferred speckle reduction method has been the filtering. Although, most of the filtering methods removed speckle noise successfully, they failed to retain the finer details related to the edges. In a medical image, the details of edges are the most important part of information in producing the exact contour of the tumor.

Directional average filters [8] are neither blurred the edges nor damaged the textual information of breast lesions while removing speckles. Anisotropic diffusion filter [9] addressed the local estimates of the image structures. In this filter, the smoothing is considered as a diffusive process and it is stopped at lesion boundaries and thus the details are preserved. The blurring of object boundaries and the loss of fine structural details are the limitations of anisotropic diffusion. Nonlinear anisotropic filter [10] achieved considerable noise reduction in images along with sharpening of boundaries. The fuzzy anisotropic diffusion filter [11], based on maximum fuzzy entropy principle mapped the input image from space domain to fuzzy domain. The fractionalorder Partial Differential Equation (PDE) is used to remove speckle noise and a sub-pixel operator is applied as tuning parameter to get optimal result. Although the diffusion based filters performed well with additive noise, they struggled with multiplicative noise. The speckle reducing anisotropic diffusion (SRAD) [12], has been developed for images with multiplicative noise. In SRAD filter, the instantaneous coefficient of variation is used as edge detector. It has demonstrated edge preserving property in breast ultrasound images by producing high values at edges and thus enhancing it and low values in homogeneous regions. However, all these filters blur the low-contrast edges and speckle noise is still retained at high-intensity regions.

Buades et al. [13] proposed the concept of Non Local Means (NLM) filter. The NLM filter exploits the repetitive patterns present in natural and texture images. It replaces each pixel in an image with a weighted average of other pixels with similar neighborhoods. The weights in the NLM filter depend on the difference of intensity values and not on the spatial distance between target patches and candidates. The NLM filter has the following advantages: 1) it is an effective way of reducing noise in highly noise corrupted images, 2) it does not affect original structures of the image such as sharp edges while removing noise and 3) it exhibits a balance between the noise attenuation and edge preservation. Both pixel-wise and block-wise NLM filers produced promising results on textured images [13]. 
However, the NLM is computationally intensive and calculation of weights in the NLM process consumes more processing time. Several improvements have been made to accelerate the processing speed [14]-[17], where dissimilar patches are eliminated before weighted averaging. Contributing neighborhoods based on mean and gradient values are preselected in [14]. In [15], Fast Fourier Transform (FFT) is employed in weights calculation. Several critical pixels in the center were utilized in weight calculation instead of all pixels in the neighborhood [16]. In [17], a strategy is presented that integrated NLM and the Laplacian pyramid to boost up the processing speed up to fifty times. However, these methods use pixels belong to a neighborhood that differs from the reference patch only by rotation or mirroring for calculating weights and the invariance under rotations and mirroring is not considered.

Grewenig et al. [18] proposed two different similarity measures; moment invariants and rotationally invariant block matching (RIBM) for NLM. The method is able to select more suitable regions for weighted averaging. In the time series NLM filter (TSNLM) [19], a speckle model related distance is used to measure the similarity between blocks and to neglect unwanted blocks. In a recent work [20], weights calculation is performed in lower dimensional subspace using principle component analysis (PCA). However, all these pre selection based methods degrade the flat regions in the image [21] which contain more similar pixels.

Yan et al. [22] proposed a NLM method which incorporated Gaussian blur, k-means clustering, and RIBM into the NLM framework. Instead of eliminating pixels during pre-selection, features are extracted from Gaussian blurred input image using moment invariants. The extracted feature vectors are clustered using k-means algorithm and they serve as Look Up Table (LUT) for noisy image. The NLM process is then performed using RIBM within each cluster instead for the entire image.

In the proposed NLM model, the Fuzzy C Means (FCM) clustering based pre classification scheme is integrated in order to ensure the selection of more suitable candidates for weighted averaging to attain better quantitative performance. The fuzzy clustering allows a candidate to be present in more than one cluster and increases the probability of selecting better candidates. The proposed model has two major advantages; firstly, the rotationally invariant moment distance measure along with the participation of all candidate pixels in NLM process increases the denoising performance and secondly, the computational time is reduced by "within cluster" weights calculation instead of the entire image.

The rest of the paper is organized as follows: Section 2 explains the details of proposed NLM algorithm. The section 3 elaborates the statistical evaluation of the proposed method using synthetic, phantom and ultrasound images. The conclusions are drawn in section 4 .

\section{METHODS}

\subsection{NLM ALGORITHM}

For a noisy image $v=\{v(i) \mid i \in \Omega\}, \Omega \subset \mathcal{R}^{2}$, the pixel intensity $N L(v)(i)$, is a weighted average of all intensity values within the neighborhood $I$. The NLM [13] is given as:

$$
N L(v)(i)=\sum_{j \in I} \omega(i, j) v(j)
$$

where, $v$ is the intensity, $v(j)$ is the intensity of pixel $j$, and $\omega(i, j)$ is the assigned weight. The value of $\omega(i, j)$ depends on the similarity between the intensities of the neighborhood blocks centered on pixel $i$ and $j$. The weights [13] are defined as:

$$
\omega_{R}(i, j)=\frac{1}{Z(i)} e^{-\frac{\left\|v N_{i}-v N_{j}\right\|_{2, a}^{2}}{h^{2}}}
$$

where, $N_{i}$ refer to a patch of fixed size and it is centered at the pixel $i$. The similarity term $\left\|v N_{i}-v N_{j}\right\|_{2, a}^{2}$ is measured as a diminishing function of the weighted Euclidean distance. The $a>$ 0 is the standard deviation of the Gaussian kernel, $Z(i)$ is the normalization constant with $Z(i)=\sum_{j} \omega(i, j)$ and $h$ is the filtering parameter.

\subsubsection{Block-wise NLM Algorithm:}

The block-wise approach of NLM [13] divides the image into overlapping blocks. The blockwise NLM reduces the computational complexity by the factor of $8[21]$. In block-wise NLM, the restoration of blocks is performed as [21]:

$$
N L(v)\left(B_{i k}\right)=\sum_{j \in I} \omega\left(B_{i k}, B_{j}\right) v\left(B_{j}\right)
$$

where, $B_{i k}$ is the block centered around pixels $i k$. For a pixel $i$ included in several blocks $B_{i k}$, several estimations of the restored intensity $N L(v)(i)$ are obtained in different $N L(v)\left(B_{i k}\right)$.

\subsection{THE PROPOSED NLM ALGORITHM}

The proposed NLM algorithm consists of following steps: 1) the input image is filtered using a median filter, 2) feature vectors are created using moment invariants 3 ) the feature vectors are clustered using FCM algorithm, 4) RIBM is performed on noisy image patches using clustered feature vectors as look up table.

\subsubsection{Preprocessing and Moment Invariants:}

Moment invariants [23] are image descriptors which are invariant under translation, changes in scale, and rotation. The input image is median filtered [30] and for an $N \times M$ image and an $n \times m$ block (centered at location $i$ ), where $=(1,2,3, \ldots, N \times M)$, the moment invariants of the patch is represented by a vector of $(1 \times 7)$. Feature vectors calculated for the entire image are the input vectors for FCM clustering. The overall process is illustrated in Fig.1.

\subsubsection{Fuzzy C Means Clustering based Pre-Classification:}

The feature vectors extracted through moment invariants are clustered using FCM. In the fuzzy based clustering, the elements of a patch are not restricted to be present in the same and single cluster; they may be spread over more than one cluster with an association defined by a membership function [24]. The objective function of FCM [25] is given as:

$$
F(\mu, a)=\sum_{i=1}^{c} \sum_{j=1}^{n} \mu_{i j}^{m}\left\|s_{j}-a_{i}\right\|^{2}
$$


where, $a_{1}, a_{2}, \ldots, a_{c}$ are the $c$ cluster centres. The $\mu_{i j}$ represents the membership of pixel $s_{j}$ in the $i^{\text {th }}$ cluster and $a_{i}$ is the cluster centre. The constant $m$ controls the fuzzyness and $m=2$ is used in this experiment. The membership functions and cluster centres are updated as:

$$
\begin{gathered}
\mu_{i j}=\frac{1}{\sum_{k=1}^{c}\left(\frac{\left\|s_{j}-a_{i}\right\|}{\left\|s_{j}-a_{k}\right\|}\right)^{\frac{2}{m-1}}}, \\
a_{i}=\frac{\sum_{i=1}^{n} \mu_{i j}^{m} s_{j}}{\sum_{i=1}^{n} \mu_{i j}^{m}}, \quad i=1,2, \ldots, c
\end{gathered}
$$

The membership function represents the probability that a pixel belongs to a specific cluster. In the FCM algorithm, the probability depends on the distance between the pixel and the cluster center [24].

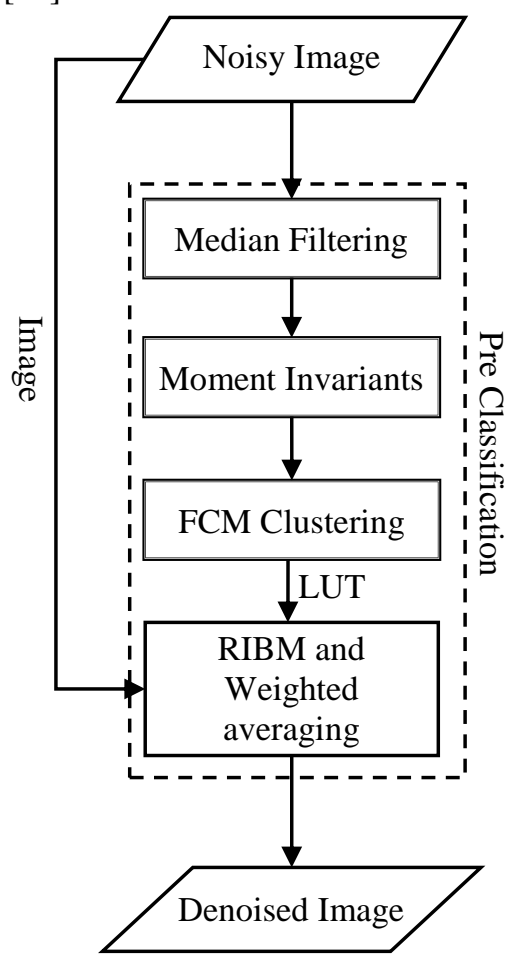

Fig.1. The proposed Method

\subsubsection{Weights Calculation Within Each Cluster:}

The weights calculation for the proposed NLM is performed within each cluster using clustering based pre-classification [22]. The function for calculating weights Eq.(3) is modified as [22]:

$$
\omega_{R}\left(B_{i k}, B_{j}\right)=\frac{1}{Z_{R}\left(B_{j}\right)} e^{-\frac{d_{R}\left(B_{i k}, B_{j}\right)}{h^{2}}}
$$

where, $\omega_{R}\left(B_{i k}, B_{j}\right)$ depends on rotationally invariant moment distance measure $d_{R}\left(B_{i k}, B_{j}\right)$ and $Z_{R}\left(B_{j}\right)=\sum_{B_{j}} \omega_{R}\left(B_{i k}, B_{j}\right)$.

\subsubsection{Rotationally Invariant Block Matching (RIBM):}

The limitation of the basic NLM algorithm is that, the lack of repetitive patterns in the image leads to unavailability of sufficient candidates for weighted averaging which degrades the denoising performance. The RIBM is a modification of the traditional block matching algorithm to find similar regions in an image which is able to detect similar patches even if they appear in several rotated or mirrored instances [18]. The centroids are used to estimate the angle of rotation between $B_{j}$; the noisy block and $B_{i k}$; the rotated (or mirrored) version of the other. The rotation matrix can be computed by finding the corresponding points in both the blocks. The corresponding point of $c_{i}$ is $c_{j}$, where the $c_{i}$ is any point in block $B_{i k}$ and $c_{j}$ is the rotated corresponding point in the block $B_{j}$. By referring the grey value of $f$ at the coordinates that are given by adding the relative coordinates $c_{i}$ to the centre of block $B_{i k}$ with $f\left(B_{i k}\right)\left(c_{i}\right)$. The modified similarity measure [22] is derived as:

$$
d_{R}\left(B_{i k}, B_{j}\right)=\int_{B_{i k}}\left(f_{B_{i k}}\left(c_{i}\right)-f_{B_{j}}\left(c_{j}\right)\right)^{2} d c_{i}
$$

Converting the equation into discrete case, we get:

$$
d_{R}\left(B_{i k}, B_{j}\right)=\int_{c_{i} \in B_{i k}}\left(f_{B_{i k}}\left(c_{i}\right)-I\left(f_{B_{j}}, c_{j}\right)\right)^{2} d c_{i}
$$

where, $I$ denotes bilinear interpolation function. For each point of $c_{i}$ in block $B_{i k}$, its corresponding point $c_{j}$ in block $B_{j}$ is obtained after rotation and interpolation.

\section{EXPERIMENTS AND DISCUSSIONS}

The proposed method is tested on standard, synthetic, phantom and real breast ultrasound images. For all test images, the proposed method is compared with other four state of the art despeckling methods such as speckle reducing anisotropic diffusion filter (SRAD) [12], anisotropic diffusion filter (ADF) [9], nonlinear anisotropic filter (NLAF) [10] and traditional NLM (TNLM)[13]. All images used in our experiments have the size of $256 \times 256$. The size of the search window is fixed at $12 \times 12$, block size is set at $7 \times 7$ and the filtering parameter $h$ is set at 15 for both NLM-based methods under comparison [13], [20], [21]. The window size of median filter is set at $5 \times 5$, moment variant $\varphi_{7}$ is used and number of clusters in FCM algorithm is set at 1200[22]. For ADF and SRAD methods, we have set number of iterations as $15, \Delta t=1 / 7$ and $\kappa=30$ [9], [12]. For Non-linear anisotropic filtering the value of $\alpha$ is set at $0.5, h=1$ and number of iterations $k=20[10]$.

All algorithms have been run on Matlab 2009a (Mathworks Inc., USA) in an Intel Core i3 processor (Intel Corp., USA) based PC with 8 GB RAM. The standard and the synthetic images are artificially added with speckle noise and simulated using Matlab. The phantom images with speckle noise are generated using Field II ultrasound simulation program [27], [28]. The real breast ultrasound images are acquired through ultrasound system (ProSound F75, Hitachi Medical Systems Europe, Switzerland) from different patients over different periods with the consent of the patients [29]. It complies with the HONcode (Health On the Net Foundation) standard for trustworthy health information with approved study protocols. 


\subsection{EXPERIMENTS ON STANDARD TEST IMAGES}

The proposed method is tested on standard images "Barbara.tif" and "Cameraman.tif" as shown in Fig.2. Speckle noise at $\sigma=0.01$ have artificially been injected using Matlab. The original image is shown in (a), (b) shows speckle added image and despeckled images using methods SRAD, ADF, NLAF, TNLM and proposed NLM method are shown in (c) to (e) respectively.

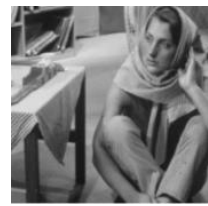

(a)

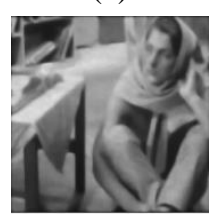

(e)

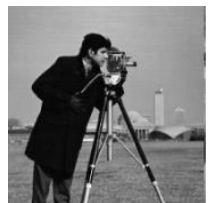

(a)

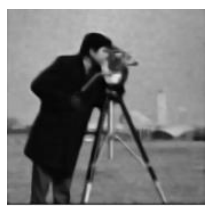

(e)

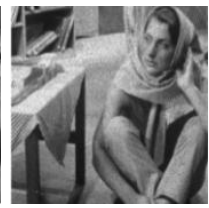

(b)

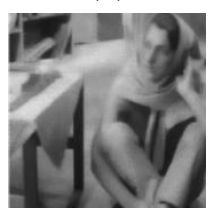

(f)

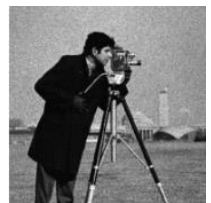

(b)

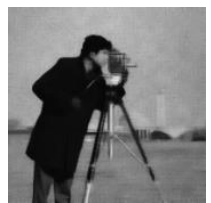

(f)

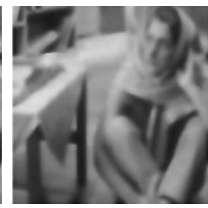

(c)

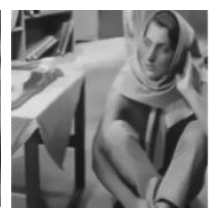

(g)

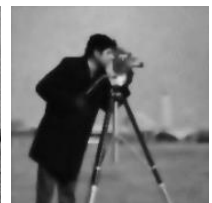

(c)

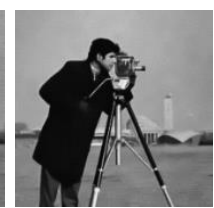

(g)

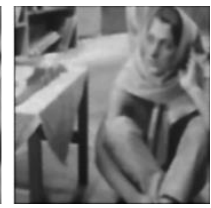

(d)
Fig.2. Despeckled results of standard test images "Barbara.tif" and "Cameraman.tif". (a) Original image, (b) Image corrupted by speckle noise $(\sigma=0.01)$, (c) to (g) despeckled images using SRAD, ADF, NLAF, TNLM and proposed NLM respectively

The statistical parameters mean squared error (MSE), peak signal to noise ratio (PSNR) [30] and structural similarity index (SSIM) [18] are used to evaluate the method. The MSE is an estimator to quantify the difference between two images, which is defined as:

$$
M S E=\frac{\sum_{i=1}^{m \times n}(\widehat{I}(m, n)-I(m, n))^{2}}{M * N}
$$

where, $M$ and $N$ are the number of rows and columns of the denoised $(\hat{I})$ and original images $(I)$. The PSNR is used to obtain the quantitative evaluations of the denoised image and it is defined as,

$$
P S N R=10 \log _{10}\left[\frac{R^{2}}{M S E}\right]
$$

where, $R$ is the maximum value of the input image data type. Since we defined them as an 8-bit unsigned integer data type, $R$ is 255 .
The SSIM expresses the similarity between denoised image and noise free image, which is calculated as,

$$
\operatorname{SSIM}(x, y)=\frac{\left(2 \mu_{I} \mu_{\hat{I}}+c_{1}\right)\left(2 \operatorname{cov}_{\hat{I}}+c_{2}\right)}{\left(\mu_{I}^{2}+\mu_{\hat{I}}^{2}+c_{1}\right)\left(\sigma_{I}^{2}+\sigma_{\hat{I}}^{2}+c_{1}\right)}
$$

where, $\mu_{I}$ and $\mu_{\hat{I}}$ are the mean of reference image and filtered image. The $\sigma_{I}^{2}$ and $\sigma_{\hat{I}}^{2}$ are the variance of reference image and filtered image respectively, the $\operatorname{cov}_{\hat{I}}$ is the covariance of filtered image. The $c_{1}, c_{2}$ are constants to avoid instability and their values are $c_{1}=0.01|\Lambda|, c_{2}=0.03|\Lambda|$ and the dynamic range of the image $|\Lambda|=255$. The Table. 1 lists the MSE, PSNR and SSIM of evaluated methods. It is shown that, the proposed method produced low MSE and high values of PSNR and SSIM.

Table.1. Lists MSE, PSNR and SSIM of all evaluated methods on standard test images corrupted by speckle noise $(\sigma=0.01)$.

Comparative values show that the proposed NLM method outperforms all other four methods in terms of MSE, PSNR and SSIM

\begin{tabular}{|c|c|c|c|c|}
\hline Test image & Method & MSE & PSNR & SSIM \\
\hline \multirow{4}{*}{ Barbara.tif } & SRAD & 266.77 & 23.48 & 0.5378 \\
\cline { 2 - 5 } & ADF & 414.68 & 21.57 & 0.6245 \\
\cline { 2 - 5 } & NLAF & 426.8 & 21.44 & 0.4826 \\
\cline { 2 - 5 } & TNLM & 183.39 & 25.14 & 0.8025 \\
\cline { 2 - 5 } & Proposed NLM & 45.13 & 31.20 & 0.9123 \\
\hline \multirow{4}{*}{ Cameraman.tif } & SRAD & 261.38 & 23.85 & 0.5637 \\
\cline { 2 - 5 } & ADF & 561.87 & 20.53 & 0.5863 \\
\cline { 2 - 5 } & TNLM & 601.06 & 20.23 & 0.4837 \\
\cline { 2 - 5 } & Proposed NLM & 58.69 & 30.36 & 0.9251 \\
\hline
\end{tabular}

The Fig. 3 shows comparative despeckling results of "Lena.tif" at different levels of speckle noise $(\sigma=0.01$ to 0.05$)$. The Table.2 lists the numeric results in terms of PSNR and SSIM. It is shown that the proposed NLM method is more robust than the ADF method in removing noise and preserving the structural similarity.

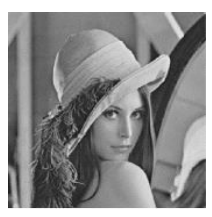

(a)

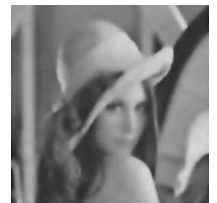

(e)

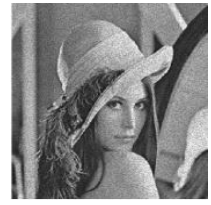

(b)

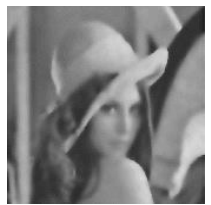

(f)

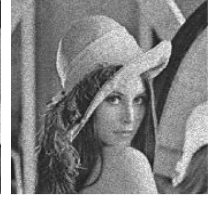

(c)

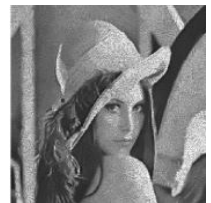

(g)

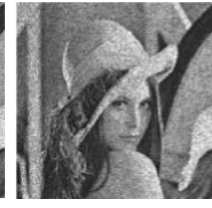

(d)

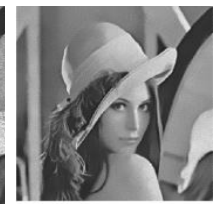

(h) 


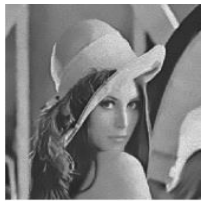

(i)

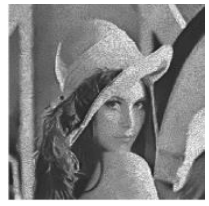

(j)
Fig.3. Despeckled results of "Lena.jpg" at different noise levels. In the first row, (a) shows original image and (b) to (d) shows speckle added images with $\sigma$ of $0.01,0.02$ and 0.05 respectively.

The second row, (e) to (f) shows despeckled images by ADF method and in the third row (h) to (j) are the despeckled images by proposed method

Table.2. Comparative denoising results of "Lena.tif" at different levels of speckle noise ( $\sigma=0.01$ to 0.05 ) by ADF method and proposed method

\begin{tabular}{|c|c|c|c|c|c|}
\hline Method & Parameters & $\boldsymbol{\sigma}=\mathbf{0 . 0 1}$ & $\boldsymbol{\sigma}=\mathbf{0 . 0 2}$ & $\boldsymbol{\sigma = 0 . 0 3}$ & $\boldsymbol{\sigma = 0 . 0 5}$ \\
\hline \multirow{2}{*}{ ADF } & PSNR & 24.38 & 23.37 & 21.43 & 19.07 \\
\cline { 2 - 6 } & SSIM & 0.7635 & 0.7138 & 0.6728 & 0.4518 \\
\hline \multirow{2}{*}{ Proposed Method } & PSNR & 31.49 & 30.67 & 28.49 & 23.94 \\
\cline { 2 - 6 } & SSIM & 0.9263 & 0.8567 & 0.7367 & 0.6528 \\
\hline
\end{tabular}

\subsection{EXPERIMENT ON SYNTHETIC IMAGE}

In this experiment, the synthetic image shown in Fig.4(a), available in MATLAB, is corrupted with simulated speckle noise of $\sigma=0.01$; based on the speckle model in Eq.(2). The speckle added image is shown in (b), despeckled images using methods using SRAD, ADF, NLAF, TNLM and proposed NLM are shown in (c) to (e) respectively.

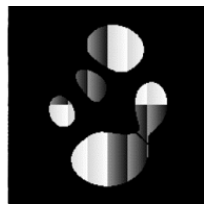

(a)

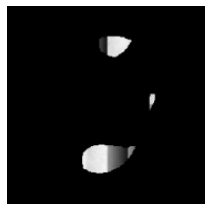

(e)

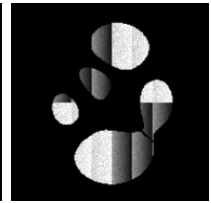

(b)

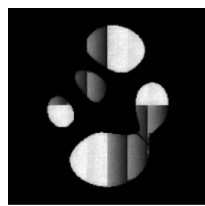

(f)

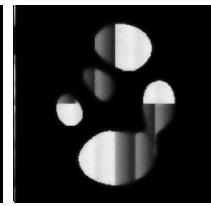

(c)

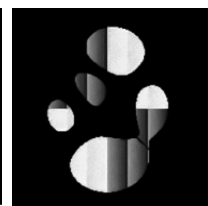

(g)

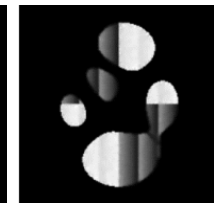

(d)
Fig.4. Despeckled results of a synthetic image. (a) Ideal image (b) Simulated speckled image with $\sigma=0.01$, (c) to (g) images filtered by SRAD, ADF, NLAF, TNLM and proposed NLM, respectively

A synthetic phantom image of head; Fig.5(a) is simulated (b) with a speckle noise of $\sigma=0.01$; the despeckling results are shown in (c) to ( $\mathrm{g}$ ) by the same set of methods. The quantitative evaluation is listed in Table.4.
Table.3. MSE, PSNR of all evaluated methods on Matlab synthetic image corrupted by speckle noise $(\sigma=0.01)$

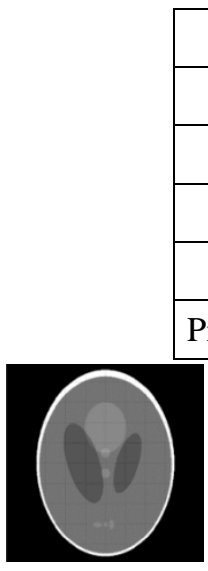

(a)

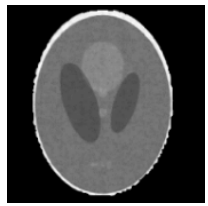

(e)

\begin{tabular}{|c|}
\hline Method \\
\hline SRAD \\
\hline ADF \\
\hline NLAF \\
\hline TNLM \\
\hline Proposed NLM \\
\hline
\end{tabular}

(b)

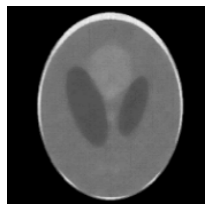

(f)

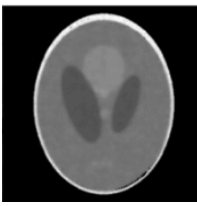

(d)
Fig.5. Despeckled results of a synthetic phantom image of head (a) Ideal image (b)Simulated speckled image with $\sigma=0.01$, (c) to $(\mathrm{g})$ images filtered by SRAD, ADF, NLAF, TNLM and proposed NLM, respectively

Table.4. MSE, PSNR of all evaluated methods on synthetic phantom image of head corrupted by speckle noise $(\sigma=0.01)$

\begin{tabular}{|c|c|c|}
\hline Method & MSE & PSNR \\
\hline SRAD & 80.51 & 29.07 \\
\hline ADF & 101.07 & 28.08 \\
\hline NLAF & 828.12 & 19.84 \\
\hline TNLM & 211.72 & 24.87 \\
\hline Proposed NLM & 13.71 & 36.76 \\
\hline
\end{tabular}

The numeric comparisons clearly indicate that the proposed method performs better than other compared methods in preserving various kinds of image features including small objects and edges. Through visual comparison, it is obvious that the ADF and SRAD blur the edges, NLAF cannot provide satisfactory speckle suppression. The TNLM method generates few minor artifacts although it can smooth out speckle effectively. By comparison, the proposed NLM method performs effective speckle noise reduction while preserving fine structures such as boundaries and weak edges effectively.

\subsection{EXPERIMENTS ON PHANTOM IMAGE}

The five evaluated despeckling methods are applied to phantom images released at http://field-ii.dk/?examples.html. To evaluate the despeckling performance of various denoising methods, we have used edge preservation index (EPI) [5] and a no-reference metric; equivalent number of looks (ENL) [19]. The EPI is defined as [5]: 


$$
E P I=\frac{\sum(\Delta S-\Delta \bar{S})(\Delta \hat{S}-\Delta \bar{S})}{\sqrt{\sum(\Delta S-\Delta \bar{S})^{2}} \sum(\Delta \hat{S}-\Delta \bar{S})^{2}}
$$

where, $\Delta S, \Delta \bar{S}$ and $\Delta \hat{S}$ are the Laplace operators of noisy image, mean image and filtered image respectively.

The inherent parameter, ENL is an effective index for estimating the speckle noise level in images. The ENL is defined as,

$$
E N L=\frac{\mu^{2}}{\sigma^{2}}
$$

where, $\mu$ is the mean and $\sigma$ is the standard deviation of the image.

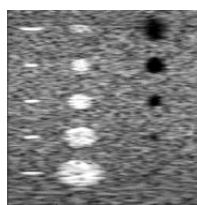

(a)

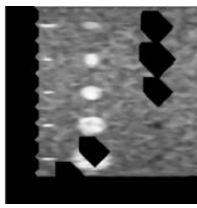

(d)

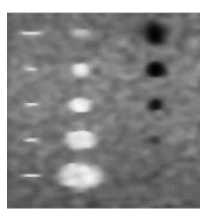

(b)

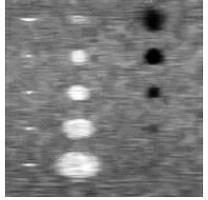

(e)

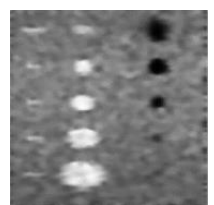

(c)

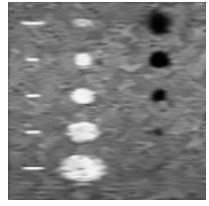

(f)
Fig.6. Comparisons of filtered results for the various methods on a cyst phantom image. (a) Original image, (b) to (f) images filtered by SRAD, ADF, NLAF, TNLM and proposed NLM respectively

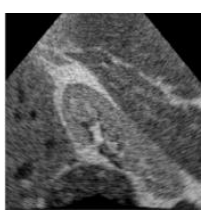

(a)

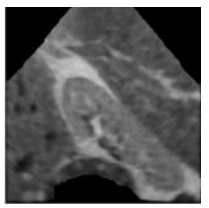

(d)

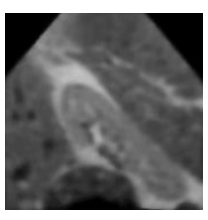

(b)

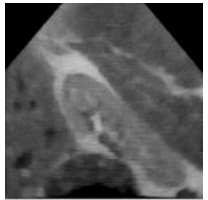

(e)

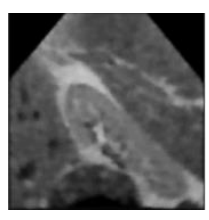

(c)

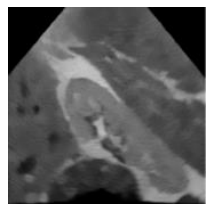

(f)
Fig.7. Comparisons of filtered results for the various methods on a phantom image of kidney (a) Original image, (b) to (f) images filtered by SRAD, ADF, NLAF, TNLM and proposed NLM respectively

As shown in Fig.6, the cyst phantom consists of finer details such as a collection of point targets, cyst regions and highly scattered regions. The Fig.7 shows a synthetic phantom of a kidney despeckled by all the methods for visual comparison. The Table.5 and Table. 6 present the values of EPI and ENL for the two different phantom images despeckled by all evaluated methods. The numeric results show that the proposed NLM method provides the highest EPI and ENL which are corresponded to better preservation of edge details.
Table.5. EPI and ENL of all evaluated methods on ultrasound cyst phantom images

\begin{tabular}{|c|c|c|}
\hline Methods & EPI & ENL \\
\hline SRAD & 0.3312 & 4.7627 \\
\hline ADF & 0.2423 & 6.0478 \\
\hline NLAF & 0.3472 & 4.1451 \\
\hline TNLM & 0.3716 & 5.3733 \\
\hline Proposed NLM & 0.3636 & 7.6079 \\
\hline
\end{tabular}

Table.6. EPI and ENL of all evaluated methods on ultrasound phantom image of kidney

\begin{tabular}{|c|c|c|}
\hline Methods & EPI & ENL \\
\hline SRAD & 0.1242 & 2.979 \\
\hline ADF & 0.1136 & 2.711 \\
\hline NLAF & 0.0712 & 1.125 \\
\hline TNLM & 0.1210 & 3.831 \\
\hline Proposed NLM & 0.2242 & 4.831 \\
\hline
\end{tabular}

\subsection{EXPERIMENTS ON REAL US IMAGES}

The proposed method is also applied to real US images to demonstrate its effectiveness. The B-mode ultrasound images of breast are used to assess the performance of the method. The comparisons among all evaluated methods on a benign cyst image are shown in Fig.8 and a malignant tumor image is shown in Fig.9. Visually, the proposed NLM method efficiently removes the speckle component while enhancing the edges and preserving fine structures in the image. The boundaries of the lesion play an important role in ultrasound based diagnosis of early breast cancer. Preserving the tiny structure of lesions is significant when they are despeckled [31]. The corresponding numerical comparisons in Table.7 show that the proposed NLM method can retain image boundaries and preserve small lesion structures more effectively than other methods.

The EPI value illustrates the effectiveness of the method in preserving the edges of the tumor. A positive and higher EPI value evidences the edge preservation capability of the filter. As listed in Table.7, the EPI values for benign and malignant categories are calculated from the individual results. Comparatively, the proposed method produced an EPI value of 0.443 on ultrasound images which is higher than the other four methods. The EPI value for malignant images are slightly lower because benign cysts have smooth and regular contour edges while the malignant tumors have irregular and rough contour edges. Although preserving edges for malignant tumors are very difficult, the proposed method has produced an EPI value of 0.429 on malignant database. The Table. 7 also shows the ENL produced on the benign and malignant databases by the all methods. A larger value of ENL corresponds to better quantitative performance of the denoising method; the proposed method clearly outperforms the other four methods. Similarly, in the same experimental setup the processing time consumed by SRAD, ADF, NLAF, TNLM and proposed NLM are 24s, 22s, 28s, 32s and 19s respectively. The better denoising performance with low processing time, make 
the proposed method more appropriate for clinical CAD systems for earlier diagnosis and characterization.

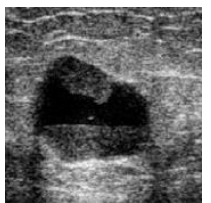

(a)

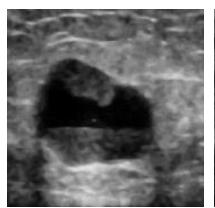

(d)

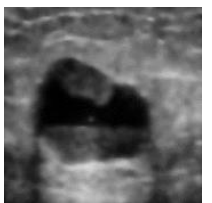

(b)

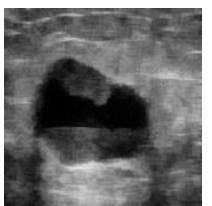

(e)

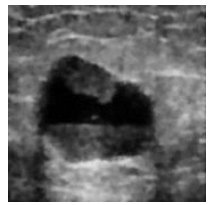

(c)

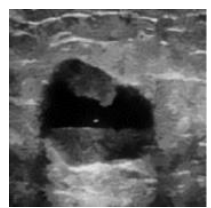

(f)
Fig.8. Despeckling results of an ultrasound breast image (benign cyst-intracystic papilloma with an intracystic hemorrhage) (a)

Original Image, (b) to (f) show the filtered images using methods SRAD, ADF, NLAF, TNLM and proposed NLM, respectively

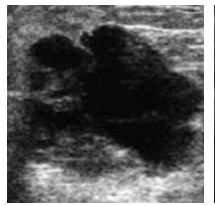

(a)

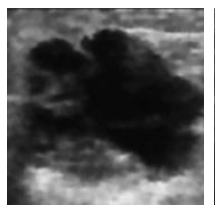

(d)

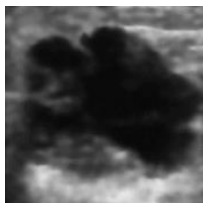

(b)

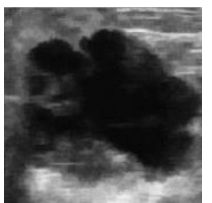

(e)

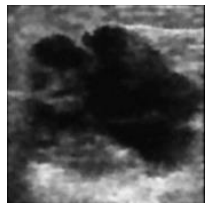

(c)

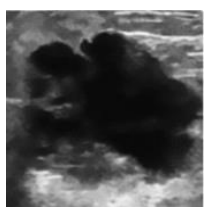

(f)
Fig.9. Despeckling results of an ultrasound breast image (malignant- Breast carcinoma with necrosis and cystic degeneration) (a) Original Image, (b) to (f) show the filtered images using methods SRAD, ADF, NLAF, TNLM and proposed NLM, respectively

Table.7. EPI and ENL values produced on benign and malignant images

\begin{tabular}{|c|c|c|c|c|c|c|}
\hline Parameters & Image Type & SRAD & ADF & NLAF & TNLM & Proposed \\
\hline \multirow{4}{*}{ EPI } & Benign (7) & 0.237 & 0.186 & 0.126 & 0.325 & 0.458 \\
\cline { 2 - 7 } & Malignant (7) & 0.216 & 0.153 & 0.092 & 0.311 & 0.429 \\
\cline { 2 - 7 } & Overall (14) & 0.226 & 0.169 & 0.109 & 0.318 & 0.443 \\
\hline \multirow{4}{*}{ ENL } & Benign (7) & 4.352 & 4.254 & 4.149 & 6.572 & 8.528 \\
\cline { 2 - 7 } & Malignant (7) & 4.143 & 4.141 & 3.983 & 6.373 & 8.163 \\
\cline { 2 - 7 } & Overall (14) & 4.247 & 4.197 & 4.066 & 6.472 & 8.345 \\
\hline
\end{tabular}

\section{CONCLUSION}

The non-local means filter is adapted for despeckling breast ultrasound images in this paper. The computational Burdon of NLM during weight calculation leads it to exhibit clumsy performance. In order to rectify the drawback, the performance of the filter has been accelerated by including FCM clustering based pre classification which also exploits moment invariants for selecting more suitable candidates for the calculation of weights. The RIBM is performed within each cluster which contains selected candidates. The denoising performance is tested by using a set of synthetic images, phantom images and on a database of real ultrasound images. The experimental results are evaluated and compared with the existing methods using five different statistical parameters to emphasize outperformance of the proposed method. The results indicated that the proposed filter is more appropriate for removing speckle noise from ultrasound images with superior edge preservation property and low computational time.

\section{ACKNOWLEDGEMENT}

We would like to acknowledge Dr. T.S.A. Geertsma, MD, Head, Department of Radiology, Gelderse Vallei Hospital, Ede, Netherlands, for providing breast ultrasound images in various categories.

\section{REFERENCES}

[1] A. Achim, A. Bezerianos and, P. Tsakalides, "Novel Bayesian Multiscale method for Speckle Removal in Medical Ultrasound Images", IEEE Transactions on Medical Imaging, Vol. 20, No. 8, pp. 772-783, 2001.

[2] K. Drukker, N.P. Gruszauskas, C.A. Sennett and M.L. Giger, "Breast US Computer-aided Diagnosis Workstation: Performance with a Large Clinical Diagnostic Population", Radiology, Vol. 248, No. 2, pp. 392-397, 2008.

[3] K.M. Prabusankarlal, P. Thirumoorthy and R. Manavalan, "Segmentation of Breast Lesions in Ultrasound Images Through Multiresolution Analysis using Undecimated Discrete Wavelet Transform”, Ultrasonic Imaging, Vol. 38, No. 6, pp. 384-402, 2016.

[4] K.M. Prabusankarlal, P. Thirumoorthy and R. Manavalan, "Computer Aided Breast Cancer Diagnosis Techniques in Ultrasound: A Survey", Journal of Medical Imaging and Health Informatics, Vol. 4, No 3, pp. 331-349, 2014.

[5] I. Njeh, O.B. Sassi, K. Chtourou and A.B. Hamida, "Speckle Noise Reduction in Breast ultrasound Images: SMU (SRAD Median Unsharp) Approach", Proceedings of $8^{\text {th }}$ IEEE International Conference on Systems, Signals and Devices, pp. 1-6, 2011.

[6] A.K. Jain, "Fundamental of Digital Image Processing", Prentice-Hall, 1989.

[7] O.V. Michailovich and A. Tannenbaum, "Despeckling of Medical Ultrasound Images", IEEE Transactions on Ultrasonics, Ferroelectrics, and Frequency, Vol. 53, No. 1, pp. 64-78, 2006.

[8] Y. Guo, H.D. Cheng, J. Tian and Y. Zhang, "A Novel approach to Speckle Reduction in Ultrasound Imaging", Ultrasound in Medicine and Biology, Vol. 35, No. 4, pp. 628-640, 2009.

[9] P. Perona and J. Malik, "Scale-Space and Edge Detection using Anisotropic Diffusion", IEEE Transactions on Pattern Analysis and Machine Intelligence, Vol. 12, No. 7, pp. 629639, 1990. 
[10] G. Gerig, O. Kubler, R. Kikinis and F.A. Jolesz, "Nonlinear Anisotropic Filtering of MRI Data", IEEE Transactions on Medical Imaging, Vol. 11, No 2, pp. 221-232, 1992.

[11] Y. Zhang, H.D. Cheng, J. Tian and J. Huang, "A Novel Speckle Reduction and Contrast Enhancement Method based on Fuzzy Anisotropic Diffusion", Proceedings of IEEE $17^{\text {th }}$ International Conference on Image Processing, pp. 4161-4164, 2011.

[12] Y. Yu and S.T. Acton, "Speckle Reducing Anisotropic Diffusion", IEEE Transactions on Image Processing, Vol. 11, No. 11, pp. 1260-1270, 2002.

[13] A. Buades, B.Coll and J.M. Morel, "A Review of Image Denoising Algorithm, with a New One", Available at: https://hal.archives-ouvertes.fr/hal-00271141/document.

[14] M. Mahmoudi and G. Sapiro, "Fast Image and Video Denoising via Nonlocal means of Similar Neighborhoods", IEEE Signal Processing Letters, Vol. 12, pp. 839-842, 2005.

[15] J. Wang, Y. Guo, Y. Ying, Y. Liu and Q. Peng, "Fast NonLocal Algorithm for Image Denoising", Proceedings of IEEE International Conference on Image Processing, pp. 1429-1432, 2006.

[16] P. Chao, O.C. Au, D. Jingjing, Y. Wen and Z. Feng, "A fast NL-means Method in Image Denoising based on the Similarity of Spatially Sampled Pixels", Proceedings of IEEE International Workshop on Multimedia Signal Processing, pp. 1-4, 2009.

[17] Y.L. Liu, J. Wang, X. Chen and Y.W. Guo, "A Robust and Fast Non-Local Means Algorithm for Image Denoising”, Journal of Computer Science and Technology, Vol. 23, No. 2, pp. 270-279, 2008.

[18] S. Grewenig, S. Zimmer and J. Weickert, "Rotationally Invariant Similarity Measures for Nonlocal Image Denoising", Journal of Visual Communication and Image Representation, Vol. 22, No. 2, pp. 117-130, 2011.

[19] Y. Gu, Z. Cui, C. Xiu and L. Wang, "Ultrasound Echocardiography Despeckling with Non-Local Means Time Series Filter", Neurocomputing, Vol. 124, pp. 120130, 2014.

[20] Y. Zhan, M. Ding, L. Wu and X. Zhang, "Nonlocal Means Method using Weight Refining for Despeckling of Ultrasound Images", Signal Processing, Vol. 103, pp. 201213, 2014..

[21] P. Coupe, P. Hellier, C. Kervrann and C. Barillot, "Nonlocal means-based Speckle Filtering for Ultrasound Images",
IEEE Transactions on Image Processing, Vol. 18, No. 10, pp. 2221-2229, 2009.

[22] R. Yan, L. Ling Shao, S.D. Cvetkovic and J. Klijn, "Improved Nonlocal means based on Pre-Classification and Invariant Block Matching”, IEEE Journal of Display Technology, Vol. 8, No. 4, pp. 212-218, 2012.

[23] M.K. Hu, "Visual Pattern by Moment Invariants", IRE Transactions on Information Theory, Vol. 8, No. 1, pp. 179187, 1962.

[24] K.S. Chuang, H.L. Tzeng, S. Chen, J. Wu and T.J. Chen, "Fuzzy C-Means Clustering with Spatial Information for Image Segmentation", Computerized Medical Imaging and Graphics, Vol. 30, pp. 9-15, 2006.

[25] K.M. Prabusankarlal, P. Thirumoorthy and R. Manavalan, "Combining Clustering, Morphology and Metaheuristic Optimization Technique for Segmentation of Breast Ultrasound Images to Detect Tumors", International Journal of Computer Applications, Vol. 86, pp. 28-34, 2014.

[26] Q. Li, H. Zhang and T. Wang, "Scale Invariant Feature Matching using Rotation-Invariant Distance for Remote Sensing Image Registration", International Journal of Pattern Recognition and Artificial Intelligence, Vol. 27, No. 2, pp. 4-22, 2013.

[27] Ultrasound Simulation Program, Available at: http:// fieldii.dk/, Accessed on 2015.

[28] J.A. Jensen and N.B. Svendsen, "Calculation of Pressure Fields from Arbitrarily Shaped, Apodized, and Excited Ultrasound Transducers", IEEE Transactions on Ultrasonics, Ferroelectrics and Frequency Control, Vol. 39, No. 2, pp. 262-267, 1992.

[29] Ultrasoundcases, Available at: http://ultrasoundcases.info/category.aspx?cat=67, Accessed on 2016

[30] K. Thangavel, R. Manavalan and I.L. Aroquiaraj, "Removal of Speckle Noise from Ultrasound Medical Image based on Special Filters: Comparative Study”, ICGST-GVIP Journal, Vol. 9, No. 3, pp. 25-32, 2009.

[31] K.M. Prabusankarlal, P. Thirumoorthy and R. Manavalan. "Classification of Breast Masses in Ultrasound Images using Self-Adaptive Differential Evolution Extreme Learning Machine and Rough Set Feature Selection", Journal of Medical Imaging, Vol. 4, No. 2, pp. 245-249, 2017. 